International Mathematical Forum, Vol. 8, 2013, no. 33, 1629 - 1640

HIKARI Ltd, www.m-hikari.com

http://dx.doi.org/10.12988/imf.2013.38156

\title{
A Note on Explicit Evaluations of Extended Euler Sums
}

\author{
Huizeng Qin ${ }^{1}$ \\ Institute of Applied Mathematics \\ Shandong University of Technology \\ Zibo, Shandong, P. R. China \\ Youmin Lu \\ Department of Mathematics and Computer Science \\ Bloomsburg University \\ Bloomsburg, PA 17815, USA
}

Copyright (c) 2013 Huizeng Qin and Youmin Lu. This is an open access article distributed under the Creative Commons Attribution License, which permits unrestricted use, distribution, and reproduction in any medium, provided the original work is properly cited.

\begin{abstract}
We study the extended Euler sums and the alternating extended Euler sums and establish their explicit expressions in terms of Riemann zeta functions and Hurwitz zeta functions. Comparing with the existing results, ours are simpler and thus yield significantly better accuracy when Matlab is used for numerical calculation.
\end{abstract}

Keywords: Extended Euler sums; Riemann zeta function; Hurwitz zeta function

\section{Introduction}

We study the extended Euler sums

$$
E_{p, q}^{(k)}=\sum_{n=1}^{\infty} \frac{1}{n^{q}} \sum_{r=1}^{k n} \frac{1}{r^{p}}, T_{p, q}^{(k)}=\sum_{n=1}^{\infty} \frac{1}{n^{q}} \sum_{r=1}^{[n / k]} \frac{1}{r^{p}}, p, q>1 .
$$

\footnotetext{
${ }^{1}$ This research was supported by NNSF number 61379009 of China.
} 
and the alternating extended Euler sums

$$
\left\{\begin{array}{c}
E_{p, q}^{(k)+,-}=\sum_{n=1}^{\infty} \frac{(-1)^{n-1}}{n^{q}} \sum_{r=1}^{k n} \frac{1}{r^{p}}, E_{p, q}^{(k)-,+}=\sum_{n=1}^{\infty} \frac{1}{n^{q}} \sum_{r=1}^{k n} \frac{\left(-1 r^{r-1}\right.}{r^{p}}, E_{p, q}^{(k)-,-}=\sum_{n=1}^{\infty} \frac{(-1)^{n-1}}{n^{q}} \sum_{r=1}^{k n} \frac{(-1)^{r-1}}{r^{p}}, \\
T_{p, q}^{(k)+,-}=\sum_{n=1}^{\infty} \frac{(-1)^{n-1}}{n^{q}} \sum_{r=1}^{[n / k]} \frac{1}{r^{p}}, T_{p, q}^{(k)-,+}=\sum_{n=1}^{\infty} \frac{1}{n^{q}} \sum_{r=1}^{[n / k]} \frac{(-1)^{r-1}}{r^{p}}, T_{p, q}^{(k)-,-}=\sum_{n=1}^{\infty} \frac{(-1)^{n-1}}{n^{q}} \sum_{r=1}^{[n / k]} \frac{(-1)^{r-1}}{r^{p}}
\end{array}\right.
$$

where $[x]$ is the largest integer $\leq x$. For $k=1$, there have been many results[1-3] about the evaluations of these sums. For $k>1, p=1$, Mark W. Coffey [4] has given a detailed study about these sums. For $k>1$, Wang and Eie [5] have developed a systematic way for establishing explicit evaluations of these sums. Our goal is to use a different way to establish expressions of these sums and improve the results by Wang and Eie. In section 3, we compare our results with Wang and Eie's and show the improvement when Matlab is used for their numerical calculations. For convenience, we write

$$
\begin{aligned}
& I_{p, q}^{(k)}=\frac{1}{2 k^{p}} \varsigma(w)+\frac{\left(1+(-1)^{q}\right)}{2} \varsigma(p) \varsigma(q)-(-1)^{q} \sum_{l=0}^{[q / 2]}\left(\begin{array}{c}
w-2 l-1 \\
p-1
\end{array}\right) k^{q-2 l} \varsigma(2 l) \varsigma(w-2 l) \\
& I_{p, q}^{(k)+-}=\frac{1}{2 k^{p}} \eta(w)+\frac{1+(-1)^{q}}{2} \eta(q) \varsigma(p)-(-1)^{q} \sum_{m=0}^{[q / 2]}\left(\begin{array}{c}
w-2 m-1 \\
p-1
\end{array}\right) k^{q-2 m} \eta(2 m) \varsigma(w-2 m), \\
& \left\{\begin{array}{c}
I_{p, q}^{(2 k)-,+}=-\frac{1}{2(2 k)^{p}} \varsigma(w)+\frac{1+(-1)^{q}}{2} \varsigma(q) \eta(p)-(-1)^{q} \sum_{m=0}^{[q / 2]}(2 k)^{q-2 m}\left(\begin{array}{c}
w-2 m-1 \\
p-1
\end{array}\right) \varsigma(2 m) \eta(w-2 m) \\
I_{p, q}^{(2 k+1)-,+}=\frac{1+(-1)^{q}}{2} \eta(p) \varsigma(q)+\frac{1}{2(2 k+1)^{p}} \eta(w)+(-1)^{q} \sum_{l=0}^{[q / 2]}\left(\begin{array}{c}
w-2 l-1 \\
p-1
\end{array}\right)(2 k+1)^{q-2 l} \eta(2 l) \eta(w-2 l)
\end{array},\right. \\
& \left\{\begin{array}{c}
I_{p, q}^{(2 k)-,-}=-\frac{1}{2(2 k)^{p}} \eta(w)+\frac{1+(-1)^{q}}{2} \eta(p) \eta(q)-(-1)^{q} \sum_{l=0}^{[q / 2]}\left(\begin{array}{c}
w-2 l-1 \\
p-1
\end{array}\right)(2 k)^{q-2 l} \eta(2 l) \eta(w-2 l) \\
I_{p, q}^{(2 k+1)-,-}=\frac{1}{2(2 k+1)^{p}} \varsigma(w)+\frac{1+(-1)^{q}}{2} \eta(p) \eta(q)+(-1)^{q} \sum_{l=0}^{[q / 2]}\left(\begin{array}{c}
w-2 l-1 \\
p-1
\end{array}\right)(2 k+1)^{q-2 l} \varsigma(2 l) \eta(w-2 l)
\end{array},\right. \\
& J_{j, k}^{(l)}=\left.\frac{(-1)^{l}}{l !} \frac{d^{l}}{d s^{l}} \cot \pi\left(s+\frac{j}{k}\right)\right|_{s=0}, K_{j, k}^{(l)}=\left.\frac{(-1)^{l}}{l !} \frac{d^{l}}{d s^{l}} \csc \pi\left(s+\frac{j}{k}\right)\right|_{s=0}, l=0,1,2, \cdots,
\end{aligned}
$$

where $\zeta(\theta)$ and $\eta(\theta)$ are the standard Riemann zeta and Dirichlet eta functions respectively.

We first summarize the main results by Wang and Eie [5]. 
Theorem A(Theorem 1[5]) For an odd weight $w=p+q, q, p>1$,

$$
E_{p, q}^{(k)}=I_{p, q}^{(k)}+(-1)^{p} k^{q-1} \sum_{r=0}^{[p / 2]}\left(\begin{array}{c}
w-2 r-1 \\
q-1
\end{array}\right) \varsigma(2 r) \varsigma(w-2 r)+C C_{p, q}^{(k)}+S S_{p, q}^{(k)},
$$

where

$$
\left\{\begin{array}{c}
C C_{p, q}^{(k)}=(-1)^{p} k^{q-1} \sum_{r=0}^{[p / 2]}\left(\begin{array}{c}
w-2 r-1 \\
q-1
\end{array}\right) \sum_{j=1}^{k-1} C_{2 r}\left(\frac{j}{k}\right) C_{w-2 r}\left(\frac{j}{k}\right) \\
S S_{p, q}^{(k)}=(-1)^{p} k^{q-1} \sum_{r=0}^{[(p-1) / 2]}\left(\begin{array}{c}
w-2 r-2 \\
q-1
\end{array}\right) \sum_{j=1}^{k-1} S_{2 r+1}\left(\frac{j}{k}\right) S_{w-2 r-1}\left(\frac{j}{k}\right)
\end{array}\right.
$$

Theorem B(Theorem 5[5]) For positive integers $m, n$, nonnegative integer $k$, and $w=$ $2 m+2 n+1$,

$$
E_{2 m, 2 n+1}^{(2 k+1)--}=\left(\begin{array}{c}
\frac{1}{2(2 k+1)^{2 m}} \varsigma(w)-\sum_{l=0}^{n}\left(\begin{array}{c}
w-2 l-1 \\
p-1
\end{array}\right)(2 k+1)^{2 n+1-2 l} \varsigma(2 l) \eta(w-2 l) \\
-(2 k+1)^{2 n} \sum_{r=0}^{m}\left(\begin{array}{c}
w-2 r-1 \\
2 n
\end{array}\right) \varsigma(2 r) \eta(w-2 r)+C C_{2 m, 2 n+1 q}^{(2 k+1)-}-S S_{2 m, 2 n+1}^{(2 k+1)-},
\end{array}\right)
$$

where

$$
\left\{\begin{array}{c}
C C_{2 m, 2 n+1}^{(k)--}=k^{2 n} \sum_{r=0}^{m}\left(\begin{array}{c}
w-2 r-1 \\
2 n
\end{array}\right) \sum_{j=1}^{k-1} C_{2 r}\left(\frac{j}{k}\right) C_{w-2 r}\left(\frac{j}{k}+\frac{1}{2}\right) \\
S S_{2 m, 2 n+1}^{(k)--}=k^{2 n} \sum_{r=0}^{m-1}\left(\begin{array}{c}
w-2 r-2 \\
2 n
\end{array}\right) \sum_{j=1}^{k-1} S_{2 r+1}\left(\frac{j}{k}\right) S_{w-2 r-1}\left(\frac{j}{k}+\frac{1}{2}\right) .
\end{array}\right.
$$

In (1.8)-(1.11), $C_{n}(\theta)$ and $S_{n}(\theta)$ are defined by

$$
C_{n}(\theta)=\sum_{l=1}^{\infty} \frac{\cos (2 l \pi \theta)}{l^{n}}, S_{n}(\theta)=\sum_{l=1}^{\infty} \frac{\sin (2 l \pi \theta)}{l^{n}}
$$

which are the cosine and the sine parts of the periodic zeta function defined by

$$
E_{n}(\theta)=\sum_{j=1}^{\infty} \frac{1}{j^{n}} e^{2 j \pi \theta i}
$$

with $S_{0}(\theta)=-\frac{1}{2}, C_{0}(\theta)=-\frac{1}{2}$.

Theorem C(Theorem 3[5]) For positive integer $n \geq 2$,

$$
E_{1,2 n}^{(k)}=\frac{2 n+1}{2 k} \varsigma(2 n+1)-\sum_{r=0}^{n-1} k^{2 n-2 r} \varsigma(2 r) \varsigma(2 n-2 r+1)-k^{2 n-1} \sum_{j=1}^{k-1} S_{1}\left(\frac{j}{k}\right) S_{2 n}\left(\frac{j}{k}\right) .
$$

The above results are clearly very beautiful, but the calculation of $C_{n}(\theta)$ and $S_{n}(\theta)$ is quite difficult. In particular, the involvement of trigonometric functions makes it very hard to ensure the accuracy of numerical calculation. On the other hand, Theorem B does not include $E_{2 m+1,2 n}^{(k)-}$ and the case when $k$ is even. To improve the results, we prove the following theorems. 
Theorem 1 For an odd weight $w=p+q$ with $p, q>1$, and $k \geq 1$, we have

$$
E_{p, q}^{(k)}=\left(\begin{array}{c}
I_{p, q}^{(k)}-\frac{(-1)^{q}}{k^{p}} \sum_{l=0}^{[p / 2]}\left(\begin{array}{c}
w-2 l-1 \\
q-1
\end{array}\right) \varsigma(2 l) \varsigma(w-2 l) \\
-\frac{(-1)^{q} \pi}{2 k^{p}} \sum_{l=0}^{p-1}\left(\begin{array}{c}
w-2-l \\
q-1
\end{array}\right) \sum_{j=1}^{k-1} J_{j, k}^{(l)} \zeta\left(w-1-l, \frac{j}{k}\right)
\end{array}\right),
$$

where $\zeta(s, a)$ is the Hurwitz zeta function defined by the formula

$$
\zeta(s, a)=\sum_{m=0}^{\infty} \frac{1}{(m+a)^{s}} .
$$

Theorem 2 For an odd weight $w=p+q$ with $p, q>1$, and $k \geq 1$, we have

$$
E_{p, q}^{(k)+,-}=\left(\begin{array}{c}
I_{p, q}^{(k)+,-}+\frac{(-1)^{q}}{k^{p}} \sum_{m=0}^{[p / 2]}\left(\begin{array}{c}
w-2 m-1 \\
q-1
\end{array}\right) \eta(2 m) \eta(w-2 m) \\
+\frac{(-1)^{q} \pi}{2 k^{p}} \sum_{l=0}^{p-1}\left(\begin{array}{c}
w-l-2 \\
q-1
\end{array}\right) \sum_{j=1}^{k-1} K_{j, k}^{(l)} \eta\left(w-1-l, \frac{j}{k}\right)
\end{array}\right)
$$

where $\eta(0)=\frac{1}{2}$ and $\eta(s, a)$ is defined by the formula

$$
\eta(s, a)=\sum_{m=0}^{\infty} \frac{(-1)^{m}}{(m+a)^{s}} .
$$

Theorem 3 For an odd weight $w=p+q$ with $p, q>1$, and $k \geq 1$, we have

$$
\left\{\begin{array}{c}
E_{p, q}^{(2 k)-,+} \\
=\left(\begin{array}{c}
I_{p, q}^{(2 k)-,+}+\frac{(-1)^{q}}{(2 k)^{p}} \sum_{m=0}^{[p / 2]}\left(\begin{array}{c}
w-2 m-1 \\
q-1
\end{array}\right) \varsigma(2 m) \varsigma(w-2 m) \\
+\frac{(-1)^{q} \pi}{2(2 k)^{p}} \sum_{l=0}^{p-1}\left(\begin{array}{c}
w-l-2 \\
q-1
\end{array}\right) \sum_{j=1}^{2 k-1}(-1)^{j} J_{j, 2 k}^{(l)} \varsigma\left(w-1-l, \frac{j}{2 k}\right)
\end{array}\right), \\
E_{p, q}^{(2 k+1)-,+}=\left(\begin{array}{c}
I^{(2 k+1)-,+}-\frac{(-1)^{q}}{(2 k+1)^{p}} \sum_{l=0}^{[p / 2]}\left(\begin{array}{c}
w-2 l-1 \\
q-1
\end{array}\right) \eta(2 l) \varsigma(w-2 l) \\
+\frac{(-1)^{q} \pi}{2(2 k+1)^{p}} \sum_{l=0}^{p-1}\left(\begin{array}{c}
w-l-2 \\
q-1
\end{array}\right) \sum_{j=1}^{2 k}(-1)^{j} K_{j, 2 k+1}^{(l)} \varsigma\left(w-1-l, \frac{j}{2 k+1}\right)
\end{array}\right) .
\end{array}\right.
$$

Theorem 4 For an odd weight $w=p+q$ with $p, q>1$, and $k \geq 1$, we have

$$
\left\{\begin{array}{c}
E_{p, q}^{(2 k)-,-}=\left(\begin{array}{c}
I_{p, q}^{(2 k)-,-}-\frac{(-1)^{q}}{(2 k)^{p}} \sum_{l=0}^{[p / 2]}\left(\begin{array}{c}
w-2 l-1 \\
q-1
\end{array}\right) \eta(2 l) \eta(w-2 l) \\
-\frac{(-1)^{q} \pi}{2(2 k)^{p}} \sum_{l=0}^{p-1}\left(\begin{array}{c}
w-l-2 \\
q-1
\end{array}\right) \sum_{j=1}^{2 k-1}(-1)^{j} K_{j, 2 k}^{(l)} \eta\left(w-1-l, \frac{j}{2 k}\right)
\end{array}\right), \\
E_{p, q}^{(2 k+1)-,-}+\frac{(-1)^{q}}{(2 k+1)^{p}} \sum_{l=0}^{[p / 2]}\left(\begin{array}{c}
w-2 l-1 \\
q-1
\end{array}\right) \varsigma(2 l) \eta(w-2 l) \\
+\frac{(-1)^{q} \pi}{2(2 k+1)^{p}} \sum_{l=0}^{p-1}\left(\begin{array}{c}
w-l-2 \\
q-1
\end{array}\right) \sum_{j=1}^{2 k}(-1)^{j-1} J_{j, 2 k+1}^{(l)} \eta\left(w-1-l, \frac{j}{k}\right)
\end{array}\right) .
$$


Theorem 5 For the integer $k \geq 1$ and $m \geq 1$, we have

$$
\begin{gathered}
E_{1,2 m}^{(k)}=\frac{2 m+1}{2 k} \varsigma(2 m+1)-\sum_{l=0}^{m-1} k^{2 m-2 l} \varsigma(2 l) \varsigma(2 m-2 l+1)-\frac{\pi}{2 k} \sum_{j=1}^{k-1} \varsigma\left(2 m, \frac{j}{k}\right) \cot \frac{j \pi}{k}, \\
E_{1,2 m}^{(k)+-}=\frac{2 m+1}{2 k} \eta(2 m+1)-\sum_{l=0}^{m-1} k^{2 m-2 l} \eta(2 l) \varsigma(2 m-2 l+1)+\frac{\pi}{2 k} \sum_{j=1}^{k-1} \eta\left(2 m, \frac{j}{k}\right) \csc \frac{j \pi}{k} \\
E^{(2 k)-+}=\left(\begin{array}{c}
-\frac{2 m+1}{4 k} \varsigma(2 m+1)-\sum_{l=1}^{m}(2 k)^{2 l} \eta(2 l+1) \varsigma(2 m-2 l) \\
+\frac{\pi}{4 k} \sum_{j=1}^{k-1}(-1)^{j} \varsigma-\left(2 m, \frac{j}{2 k}\right) \cot \frac{j \pi}{2 k} \\
E^{(2 k+1)-+}=\left(\begin{array}{c}
-\frac{m}{2 k+1} \varsigma(2 m+1)+\frac{1}{4 k+2} \eta(2 m+1)+\sum_{l=1}^{m}(2 k+1)^{2 l} \eta(2 l+1) \eta(2 m-2 l) \\
1,2 m
\end{array}\right) \\
+(\varsigma(2 m)+\eta(2 m)) \ln 2+\frac{\pi}{4 k+2} \sum_{j=1}^{k}(-1)^{j} \varsigma_{-}\left(2 m, \frac{j}{2 k+1}\right) \csc \frac{j \pi}{2 k+1}
\end{array}\right)
\end{gathered}
$$

and

$$
\left\{\begin{array}{c}
E_{1,2 m}^{(2 k)--}=\left(\begin{array}{c}
-\frac{2 m+1}{4 k} \eta(2 m+1)-\sum_{l=1}^{m}(2 k)^{2 l} \eta(2 l+1) \eta(2 m-2 l) \\
+\frac{\pi}{4 k}(-1)^{k-1} \eta\left(2 m, \frac{1}{2}\right)+\frac{\pi}{4 k} \sum_{j=1}^{k-1}(-1)^{j-1} \eta_{+}\left(2 m, \frac{j}{2 k}\right) \csc \frac{j \pi}{2 k}
\end{array}\right), \\
E_{1,2 m}^{(2 k+1)--}=\left(\begin{array}{c}
\frac{\varsigma(2 m+1)}{4 k+2}-\frac{m}{2 k+1} \eta(2 m+1)+\sum_{l=1}^{m}(2 k+1)^{2 l} \eta(2 l+1) \varsigma(2 m-2 l) \\
+(\varsigma(2 m)+\eta(2 m)) \ln 2+\frac{\pi}{4 k+2} \sum_{j=1}^{k}(-1)^{j-1} \eta_{+}\left(2 m, \frac{j}{2 k+1}\right) \cot \frac{j \pi}{2 k+1}
\end{array}\right) .
\end{array}\right.
$$

Remark 6 (1.9) requires $k$ to be odd. Theorems 2-4 have released this requirement. Theorem B is about $E_{2 m, 2 n+1}^{(2 k+1)-}$ and $E_{2 m+1,2 n}^{(2 k+1)-}$ is not included. In fact, Theorem 1 solves this problem.

$$
E_{2 m+1,2 n}^{(2 k+1)--}=\left(\begin{array}{c}
\eta(2 m+1) \varsigma(2 n)+\frac{1}{(2 k+1)^{2 m}} \sum_{l=0}^{m}\left(\begin{array}{c}
w-2 l-1 \\
q-1
\end{array}\right) \varsigma(2 l) \eta(w-2 l) \\
+\frac{1}{2(2 k+1)^{2 m+1}} \varsigma(w)+\sum_{l=0}^{n}\left(\begin{array}{c}
w-2 l-1 \\
p-1
\end{array}\right)(2 k+1)^{2 n-2 l} \varsigma(2 l) \eta(w-2 l) \\
-\frac{\pi}{2(2 k+1)^{2 m+1}} \sum_{l=0}^{2 m} \frac{(-1)^{l}}{l !}\left(\begin{array}{c}
w-l-2 \\
q-1
\end{array}\right)\left(\sum_{j=1}^{2 k}-1\right)^{j} J_{j, 2 k+1}^{l} \eta\left(w-1-l, \frac{j}{k}\right)
\end{array}\right) .
$$


Remark 7 Comparing Theorems A-C and Theorems 1-5, we may obtain a group of identities some of which may be new. For example, Theorems $A$ and 1 give the following identity.

$$
\begin{aligned}
C C_{p, q}^{(k)}+S S_{p, q}^{(k)}= & \left((-1)^{q+1} k^{-p}+(-1)^{p+1} k^{q-1}\right) \sum_{l=0}^{[p / 2]}\left(\begin{array}{c}
w-2 l-1 \\
q-1
\end{array}\right) \zeta(2 l) \zeta(w-2 l) \\
& -\frac{(-1)^{q} \pi}{2 k^{p}} \sum_{l=0}^{p-1}\left(\begin{array}{c}
w-l-2 \\
q-1
\end{array}\right) \sum_{j=1}^{k-1} J_{j, k}^{(l)} \zeta\left(w-l-1, \frac{j}{k}\right) .
\end{aligned}
$$

\section{Proof of Theorems}

We use the classical residue method used by $[1,2]$. The following lemma plays an important role in the proof as it does in the calculation of all other Euler sums.

Lemma 8 (Cauchy, Lindelöf [2]) Let $\xi(s)$ be a kernel function and $r(s)$ be a rational function which is $O\left(s^{-2}\right)$ at infinity. Then

$$
\left.\sum_{\alpha \in O} \operatorname{Res}(r(s) \xi(s))\right|_{s=\alpha}=-\left.\sum_{\beta \in S} \operatorname{Res}(r(s) \xi(s))\right|_{s=\beta}
$$

where $S$ is the set of poles of $r(s), O$ is the set of the poles of $r(s)$ that are not poles of $\xi(s)$ and $\left.\operatorname{Res}(h(s))\right|_{s=\lambda}$ denotes the residue of $h(s)$ at $\lambda$.

The key of using the residue method is the wise selection of kernel functions. Here, we make an essential use of kernels

$$
\psi^{ \pm}(k, s)=\frac{1}{k s}+\sum_{m=1}^{\infty}( \pm 1)^{m}\left(\frac{1}{m}-\frac{1}{m-k s}\right), \cot \pi s, \text { and } \csc \pi s .
$$

The poles of $\psi^{ \pm}(k, s)$ are at the integers $n$ and fractions $n+\frac{j}{k}$ with $j=1,2, \cdots, k-1$ and $n=0,1,2, \cdots$. The power series expansions of $\psi^{ \pm}(k, s)$ at these poles are, respectively,

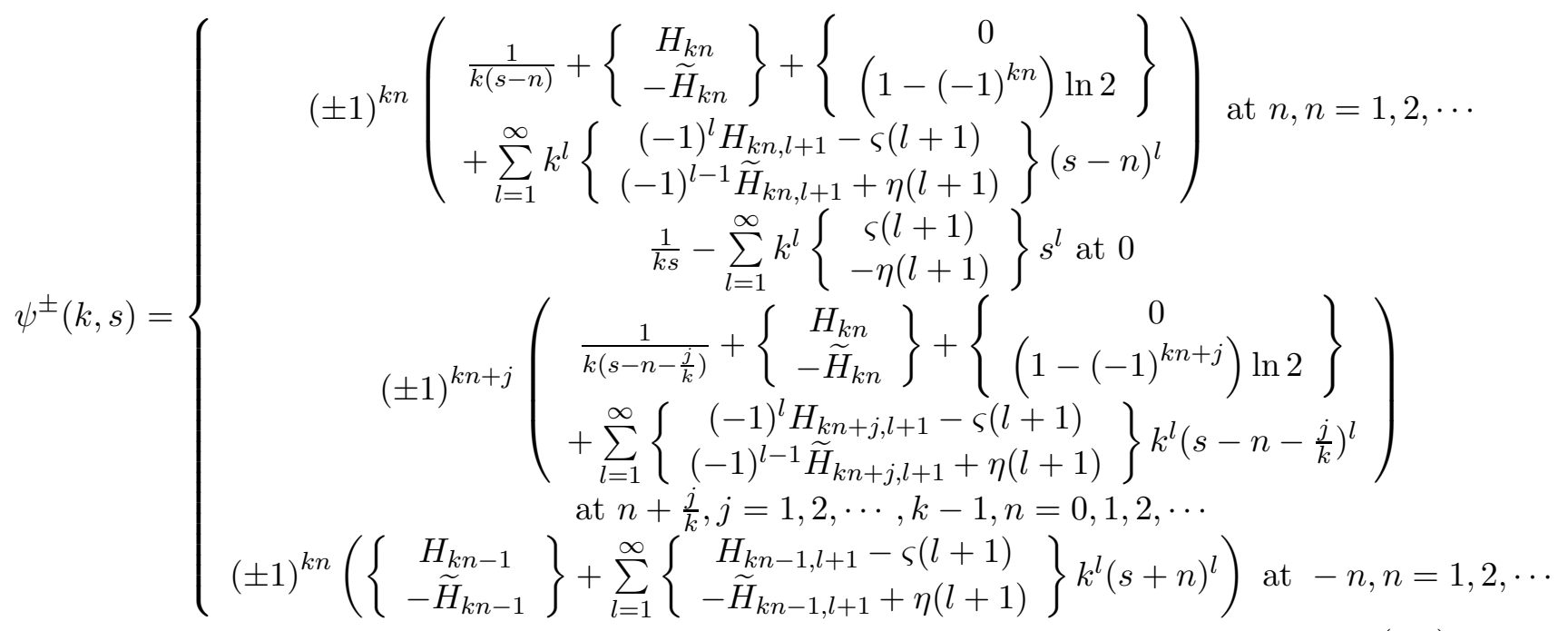


where

$$
H_{n}=H_{n, 1}, H_{n, p}=\sum_{l=1}^{n} \frac{1}{l^{p}}, \widetilde{H}_{n}=\widetilde{H}_{n, 1}, \text { and } \widetilde{H}_{n, p}=\sum_{j=1}^{n} \frac{(-1)^{j-1}}{j^{p}} .
$$

By (2.3), we have

$$
\left\{\begin{array}{c}
\frac{1}{(p-1) !} \frac{d^{p-1}}{d s^{p-1}} \psi^{ \pm}(k, s)= \\
( \pm 1)^{k n}\left(\frac{(-1)^{p-1}}{k(s-n)^{p}}+\sum_{l=0}^{\infty}\left(\begin{array}{c}
l+p-1 \\
p-1
\end{array}\right) k^{l+p-1}\left\{\begin{array}{c}
(-1)^{l+p-1} H_{k n, l+p}-\varsigma(l+p) \\
(-1)^{l+p} \widetilde{H}_{k n, l+p}+\eta(l+p)
\end{array}\right\}(s-n)^{l}\right) \\
\text { at } n=1,2, \cdots \\
\frac{(-1)^{p-1}}{k s^{p}}-\sum_{l=0}^{\infty}\left(\begin{array}{c}
l+p-1 \\
p-1
\end{array}\right) k^{l+p-1}\left\{\begin{array}{c}
\varsigma(l+p) \\
-\eta(l+p)
\end{array}\right\} s^{l} \text { at } 0 \\
( \pm 1)^{k n+j}\left(\begin{array}{c}
\left.\frac{(-1)^{p-1}}{k\left(s-n-\frac{j}{k}\right)^{p}}+\sum_{l=0}^{\infty}\left(\begin{array}{c}
l+p-1 \\
p-1
\end{array}\right)\left\{\begin{array}{c}
(-1)^{l+p-1} H_{k n+j, l+p}-\varsigma(l+p) \\
(-1)^{l+p} \widetilde{H}_{k n+j, l+p}+\eta(l+p)
\end{array}\right\} k^{l+p-1}\left(s-n-\frac{j}{k}\right)^{l}\right) \\
\text { at } n+\frac{j}{k}, j=1,2, \cdots, k-1, n=0,1,2, \cdots \\
( \pm 1)^{k n} \sum_{l=0}^{\infty}\left(\begin{array}{c}
l+p-1 \\
p-1
\end{array}\right) k^{l+p-1}\left\{\begin{array}{c}
H_{k n-1, l+p}-\varsigma(l+p) \\
-\widetilde{H}_{k n-1, l+p}+\eta(l+p)
\end{array}\right\}(s+n)^{l} \text { at }-n, n=1,2, \cdots
\end{array}\right.
\end{array}\right.
$$

The kernels $\pi \cot \pi s$ and $\pi \csc \pi s$ have the following expansions, respectively,

$$
\pi \cot \pi s=\frac{1}{s-n}-2 \sum_{m=0}^{\infty} \varsigma(2 m+2)(s-n)^{2 m+1} \text { at } n=0, \pm 1, \pm 2, \cdots
$$

and

$$
\pi \csc \pi s=(-1)^{n}\left(\frac{1}{s-n}+2 \sum_{m=0}^{\infty} \eta(2 m+2)(s-n)^{2 m+1}\right) \text { at } n=0, \pm 1, \pm 2, \cdots
$$

Consequently, the residues of these functions are

$$
\left\{\begin{array}{l}
\mathrm{R} \\
\mathrm{R}
\end{array}\right.
$$$$
\operatorname{Res}\left[\frac{\pi \cot \pi s}{(p-1) ! s^{q}} \frac{d^{p-1}}{d s^{p-1}} \psi^{ \pm}(k, s), n\right]=( \pm 1)^{k n}\left(\begin{array}{c}
+\frac{k^{p-1}}{n^{q}}\left\{\begin{array}{c}
(-1)^{p-1} H_{k n, p}-\varsigma(p) \\
(-1)^{p} \widetilde{H}_{k n, p}+\eta(p)
\end{array}\right\} \\
+\frac{2}{k} \sum_{m=0}^{[p / 2]} \varsigma(2 m)\left(\begin{array}{c}
w-2 m-1 \\
q-1
\end{array}\right) \frac{1}{n^{w-2 m}}
\end{array}\right)
$$$$
\text { for } n=1,2, \cdots
$$

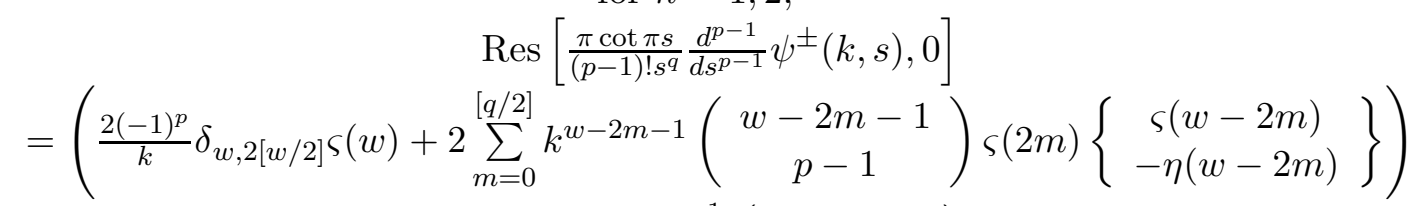

$\operatorname{Res}\left[\frac{\pi \cot \pi s}{(p-1) ! s^{q}} \frac{d^{p-1}}{d s^{p-1}} \psi^{ \pm}(k, s), n+\frac{j}{k}\right]=\left.\frac{( \pm 1)^{k n+j} \pi}{k} \sum_{l=0}^{p-1}\left(\begin{array}{c}w-l-2 \\ q-1\end{array}\right) \frac{(-1)^{l}}{l !\left(n+\frac{j}{k}\right)^{w-1-l}} \frac{d^{l}}{d s^{l}} \cot \pi\left(s+\frac{j}{k}\right)\right|_{s=0}$, for $j=1,2, \cdots, k-1, n=0,1,2, \cdots$

$\operatorname{Res}\left[\frac{\pi \cot \pi s}{(p-1) ! s^{q}} \frac{d^{p-1}}{d s^{p-1}} \psi^{ \pm}(k, s),-n\right]=(-1)^{q} \frac{( \pm 1)^{k n} k^{p-1}}{n^{q}}\left\{\begin{array}{c}H_{k n-1, p}-\varsigma(p) \\ -\widetilde{H}_{k n-1, p}+\eta(p)\end{array}\right\}, n=1,2, \cdots$. 
and

$$
\begin{aligned}
& \operatorname{Res}\left[\frac{\pi \csc \pi s}{(p-1) ! s^{q}} \frac{d^{p-1}}{d s^{p-1}} \psi^{ \pm}(k, s), n\right]=( \pm 1)^{k n}(-1)^{n}\left(\begin{array}{c}
\frac{1}{n^{q}} k^{p-1}\left\{\begin{array}{c}
(-1)^{p-1} H_{k n, p}-\varsigma(p) \\
(-1)^{p} \widetilde{H}_{k n, p}+\eta(p)
\end{array}\right. \\
-\frac{2}{k} \sum_{m=0}^{[p / 2]}\left(\begin{array}{c}
w-2 m-1 \\
q-1
\end{array}\right) \eta(2 m) \frac{1}{n^{w-2 m}}
\end{array}\right) \\
& \text { for } n=1,2, \cdots \text {, } \\
& \operatorname{Res}\left[\frac{\pi \csc \pi s}{(p-1) ! s^{q}} \frac{d^{p-1}}{d s^{p-1}} \psi^{ \pm}(k, s), 0\right]= \\
& \left(\frac{2(-1)^{p-1}}{k} \delta_{w, 2[w / 2]} \eta(w)-2 \sum_{m=0}^{[q / 2]}\left(\begin{array}{c}
w-2 m-1 \\
p-1
\end{array}\right) k^{w-2 m-1} \eta(2 m)\left\{\begin{array}{c}
\varsigma(w-2 m) \\
-\eta(w-2 m)
\end{array}\right\}\right), \\
& \operatorname{Res}\left[\frac{\pi \csc \pi s}{(p-1) ! s^{q}} \frac{d^{p-1}}{d s^{p-1}} \psi^{ \pm}(k, s), n+\frac{j}{k}\right]=\left.\frac{( \pm 1)^{k n+j} \pi}{k} \sum_{l=0}^{p-1}\left(\begin{array}{c}
w-l-2 \\
q-1
\end{array}\right) \frac{(-1)^{n}}{\left(n+\frac{j}{k}\right)^{w-1-l}} \frac{(-1)^{l}}{l !} \frac{d^{l}}{d s^{l}} \csc \pi\left(s+\frac{j}{k}\right)\right|_{s=0} \\
& \text { for } j=1,2, \cdots, k-1, n=0,1,2, \cdots \\
& \operatorname{Res}\left[\frac{\pi \csc \pi s}{(p-1) ! s^{q}} \frac{d^{p-1}}{d s^{p-1}} \psi^{ \pm}(k, s),-n\right]=(-1)^{q+n} \frac{( \pm 1)^{k n} k^{p-1}}{n^{q}}\left\{\begin{array}{c}
H_{k n-1, p}-\varsigma(p) \\
-\widetilde{H}_{k n-1, p}+\eta(p)
\end{array}\right\}, n=1,2, \cdots
\end{aligned}
$$

Applying kernel functions $\xi(s)=\frac{\pi \cot \pi s}{(p-1) !} \frac{d^{p-1}}{d s^{p-1}} \psi^{ \pm}(k, s), \frac{\pi \csc \pi s}{(p-1) !} \frac{d^{p-1}}{d s^{p-1}} \psi^{ \pm}(k, s)$ to base function $r(s)=s^{-q}$ respectively, we can obtain theorems 1-4 by using Lemma 1 and results (2.8) and (2.9). Similarly, applying kernel functions $\xi(s)=\pi \cot \pi s \psi^{ \pm}(k, s), \pi \csc \pi s \psi^{ \pm}(k, s)$ to the base function $r(s)=s^{-q}$ respectively, one may obtain theorem 5 .

\section{Simplification of Theorems A-C and Numerical Com- parisons}

As mentioned in section 1, the involvement of trigonometric functions in Theorems A-C causes larger error in numerical calculation. To compare our results with Theorems A-C, we first simplify Theorems A-C and try to eliminate the trigonometric functions. We will use the following standard notations for $\varsigma_{ \pm}(q, x)$ and $\eta_{ \pm}(q, x)$.

$$
\begin{gathered}
\varsigma_{+}(q, x)=\varsigma(q, x)+\varsigma(q, 1-x), \varsigma_{-}(q, x)=\varsigma(q, x)-\varsigma(q, 1-x), \\
\eta_{+}(q, x)=\eta(q, x)+\eta(q, 1-x), \eta_{-}(q, x)=\eta(q, x)-\eta(q, 1-x), K_{m, k}=\sum_{j=1}^{k-1} j \sin \frac{2 m \pi j}{k} .
\end{gathered}
$$

It is well-known that the Hurwitz zeta function and the trigonometric functions are related by the identities:

$$
\varsigma(n, 1-x)+(-1)^{n} \varsigma(n, x)=\frac{(2 \pi i)^{n}}{2(n-1) !}(i \cot \pi x+1) \sum_{k=1}^{n-1} \frac{k !}{2^{k}}\left\{\begin{array}{c}
n-1 \\
k
\end{array}\right\}(i \cot \pi x-1)^{k},
$$

where $\left\{\begin{array}{l}j \\ k\end{array}\right\}$ is the Stirling subset number, and

$$
\varsigma\left(2 n+1,1-\frac{p}{q}\right)-\varsigma\left(2 n+1, \frac{p}{q}\right)=-\frac{\pi^{2 n+1}}{(2 n) !} \lim _{y \rightarrow 0} \frac{d^{2 n}}{d y^{2 n}} \frac{\sin (2 \pi p / q)}{\cos (2 y)-\cos (2 \pi p / q)} .
$$


By using Fourier expansion, we know

$$
S_{1}(\theta)=\sum_{l=1}^{\infty} \frac{\sin (2 l \pi \theta)}{l}=\pi\left(\frac{1}{2}-\theta\right), 0<\theta<1 .
$$

We can rewrite $C_{n}(\theta)$ as

$$
C_{n}\left(\frac{j}{k}\right)=\sum_{l=0}^{\infty} \sum_{m=1}^{k} \frac{1}{(k l+m)^{n}} \cos \frac{2 m \pi j}{k}=\frac{1}{k^{n}}\left(\sum_{m=1}^{k-1} \varsigma\left(n, \frac{m}{k}\right) \cos \frac{2 m \pi j}{k}+\varsigma(n)\right) .
$$

Similarly we may write $S_{n}(\theta)$ and $C_{n}\left(\frac{j}{k}+\frac{1}{2}\right)$ as

$$
\begin{gathered}
C_{n}\left(\frac{j}{2 k}+\frac{1}{2}\right)=\frac{1}{(2 k)^{n}}\left(\sum_{m=1}^{2 k-1}(-1)^{m} \varsigma\left(n, \frac{m}{2 k}\right) \cos \frac{m \pi j}{k}+\varsigma(n)\right), \\
C_{n}\left(\frac{j}{2 k+1}+\frac{1}{2}\right)=\frac{1}{(2 k+1)^{n}}\left(\sum_{m=1}^{2 k}(-1)^{m} \eta\left(n, \frac{m}{2 k+1}\right) \cos \frac{2 m \pi j}{2 k+1}-\eta(n)\right), \\
S_{n}\left(\frac{j}{k}\right)=\frac{1}{k^{n}} \sum_{m=1}^{k-1} \varsigma\left(n, \frac{m}{k}\right) \sin \frac{2 m \pi j}{k}, \\
S_{n}\left(\frac{j}{2 k}+\frac{1}{2}\right)=\frac{1}{(2 k)^{n}} \sum_{m=1}^{2 k-1}(-1)^{m} \varsigma\left(n, \frac{m}{2 k}\right) \sin \frac{m \pi j}{k}, \\
S_{n}\left(\frac{j}{2 k+1}+\frac{1}{2}\right)=\frac{1}{(2 k+1)^{n}} \sum_{m=1}^{2 k}(-1)^{m} \eta\left(n, \frac{m}{2 k+1}\right) \sin \frac{2 m \pi j}{2 k+1} .
\end{gathered}
$$

Using the properties of the Hurwitz zeta function

$$
\sum_{l=1}^{k-1} \varsigma\left(q, \frac{l}{k}\right)=\left(k^{q}-1\right) \varsigma(q)
$$

and

$$
\sum_{j=1}^{k-1} \cos \frac{2 m \pi j}{k}=-1, \sum_{j=1}^{k-1} \sin \frac{2 m \pi j}{k}=0,1<m<k, k>1
$$

one may get

$$
\begin{gathered}
\sum_{j=1}^{k-1} C_{n}\left(\frac{j}{k}\right)=\left(\frac{1}{k^{n-1}}-1\right) \varsigma(n), \\
\sum_{j=1}^{2 k} C_{n}\left(\frac{j}{2 k+1}+\frac{1}{2}\right)=\frac{1}{(2 k+1)^{n}}\left(\sum_{m=1}^{k}(-1)^{m-1} \eta_{-}\left(n, \frac{m}{2 k+1}\right)-2 k \eta(n)\right), \\
\sum_{j=1}^{k-1} S_{n}\left(\frac{j}{k}\right)=0, \sum_{j=1}^{k-1} S_{n}\left(\frac{j}{k}+\frac{1}{2}\right)=0 .
\end{gathered}
$$

By (3.3),(3.4),(3.6) and

$$
\begin{aligned}
& \sum_{j=1}^{k-1} \cos \frac{2 m \pi j}{k} \cos \frac{2 l \pi j}{k}=\frac{k}{2} \delta_{m, l}+\frac{k}{2} \delta_{m, k-l}-1,0<m, l<k \\
& \sum_{j=1}^{k-1} \sin \frac{2 m \pi j}{k} \sin \frac{2 l \pi j}{k}=\frac{k}{2} \delta_{m, l}-\frac{k}{2} \delta_{m, k-l}, 0<m, l<k
\end{aligned}
$$


we have

$$
\begin{gathered}
\sum_{j=1}^{k-1} C_{2 r}\left(\frac{j}{k}\right) C_{w-2 r}\left(\frac{j}{k}\right)=\left(\frac{1}{k^{w-1}}-1\right) \varsigma(2 r) \varsigma(w-2 r)+\frac{1}{2 k^{w-1}} \sum_{m=1}^{k-1} \varsigma\left(2 r, \frac{m}{k}\right) \varsigma_{+}\left(w-2 r, \frac{m}{k}\right), \\
\sum_{j=1}^{2 k} C_{2 r}\left(\frac{j}{2 k+1}\right) C_{w-2 r}\left(\frac{j}{2 k+1}+\frac{1}{2}\right)=\frac{1}{(2 k+1)^{w-1}}\left(\begin{array}{c}
(2 k+1)^{2 r-1} \varsigma(2 r) \sum_{m=1}^{k}(-1)^{m-1} \eta_{-}\left(w-2 r, \frac{m}{2 k+1}\right) \\
+\frac{1}{2} \sum_{m=1}^{k}(-1)^{m} \varsigma_{+}\left(2 r, \frac{m}{2 k+1}\right) \eta_{-}\left(w-2 r, \frac{m}{2 k+1}\right) \\
+\left((2 k+1)^{2 r-1}-1\right) \varsigma(2 r) \eta(w-2 r)
\end{array}\right), \\
\sum_{j=1}^{k-1} S_{1}\left(\frac{j}{k}\right) S_{w-1}\left(\frac{j}{k}\right)=-\frac{\pi}{k^{w}} \sum_{m=1}^{k-1} K_{m, k} \varsigma\left(w-1, \frac{m}{k}\right) \\
\sum_{j=1}^{2 k} S_{1}\left(\frac{j}{2 k+1}\right) S_{w-1}\left(\frac{j}{2 k+1}+\frac{1}{2}\right)=\frac{\pi}{(2 k+1)^{w}} \sum_{l=1}^{2 k}(-1)^{l+1} K_{l, 2 k+1} \eta\left(w-1, \frac{l}{2 k+1}\right) \\
\sum_{j=1}^{k-1} S_{2 r+1}\left(\frac{j}{k}\right) S_{w-2 r-1}\left(\frac{j}{k}\right)=\frac{1}{2 k^{w-1}} \sum_{m=1}^{k-1} \varsigma\left(2 r+1, \frac{m}{k}\right) \varsigma-\left(w-2 r-1, \frac{m}{k}\right), \\
\sum_{j=1}^{2 k} S_{2 r+1}\left(\frac{j}{2 k+1}\right) S_{w-2 r-1}\left(\frac{j}{2 k+1}+\frac{1}{2}\right)=\frac{1}{2(2 k+1)^{w-1}} \sum_{m=1}^{2 k}(-1)^{m} \varsigma\left(2 r+1, \frac{m}{2 k+1}\right) \eta_{+}\left(w-2 r-1, \frac{m}{2 k+1}\right)
\end{gathered}
$$

Substituting (3.2) and (3.7) into (1.9), (1.11) and (1.14), we get

$$
\left\{\begin{array}{c}
C C_{p, q}^{(k)}=\left(\begin{array}{c}
\left(\frac{1}{k^{p}}-k^{q-1}\right)(-1)^{p} \sum_{r=0}^{[p / 2]}\left(\begin{array}{c}
w-2 r-1 \\
q-1
\end{array}\right) \varsigma(2 r) \varsigma(w-2 r) \\
+\frac{(-1)^{p}}{2 k^{p}} \sum_{r=1}^{[p / 2]}\left(\begin{array}{c}
w-2 r-1 \\
q-1
\end{array}\right) \sum_{m=1}^{k-1} \varsigma\left(2 r, \frac{m}{k}\right) \varsigma_{+}\left(w-2 r, \frac{m}{k}\right)
\end{array}\right) \\
-\frac{(-1)^{p} \pi}{k^{p+1}}\left(\begin{array}{c}
w-2 \\
q-1
\end{array}\right) \sum_{m=1}^{k-1} K_{m, k} \varsigma\left(w-1, \frac{m}{k}\right) \\
S S_{p, q}^{(k)}=\left(\begin{array}{c}
w-2 r-2 \\
q-1
\end{array}\right) \sum_{m=1}^{k-1} \varsigma\left(2 r+1, \frac{m}{k}\right) \varsigma_{-}\left(w-2 r-1, \frac{m}{k}\right)
\end{array}\right),
$$

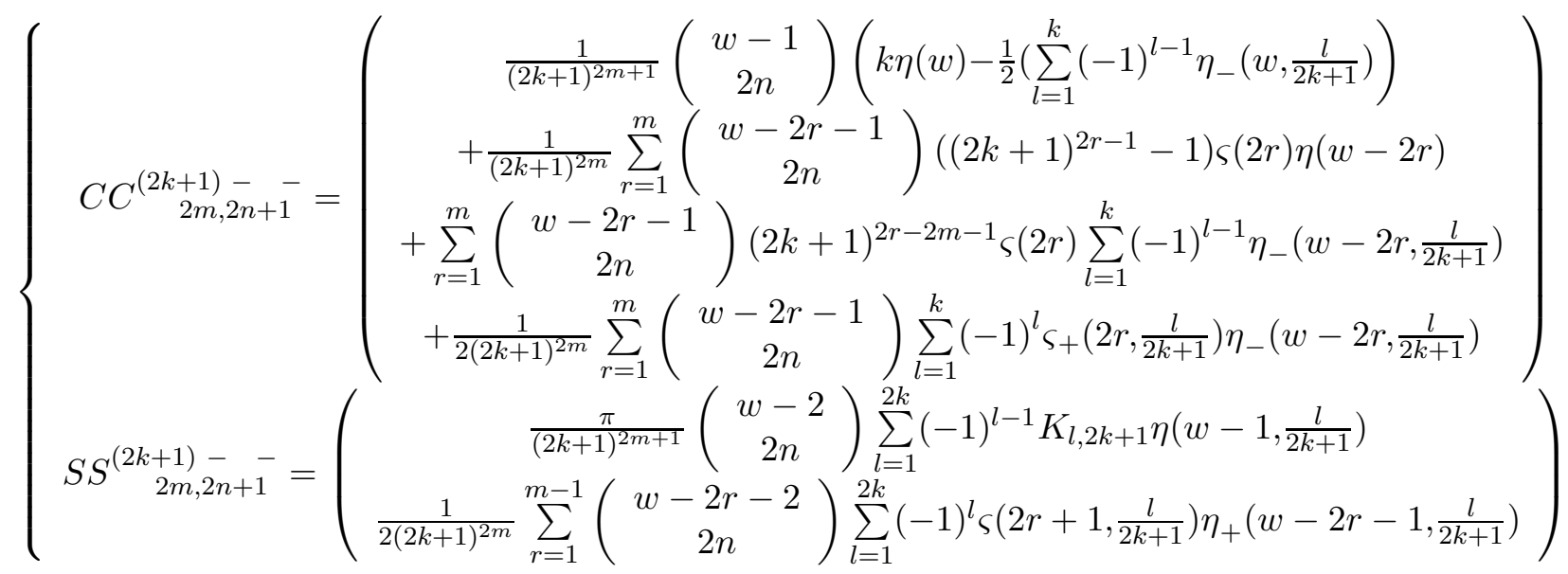


and

$$
E_{1,2 n}^{(k)}=\frac{2 n+1}{2 k} \varsigma(2 n+1)-\sum_{l=0}^{n-1} k^{2 n-2 r} \varsigma(2 r) \varsigma(2 n-2 r+1)+\frac{\pi}{k^{2 m}} \sum_{m=1}^{k-1} K_{m, k} \varsigma\left(2 n, \frac{m}{k}\right) .
$$

Now, we compare the efficiency of the expressions given by Theorems A-C, Theorems 1-5 and those obtained in this section. The following table shows the numerical results and the errors for the calculation of $E_{1,2 n}^{(k)}$ by using Matlab.

\begin{tabular}{cccccc}
\multicolumn{5}{c}{ Error Comparison for } & $(1.14),(3.10)$ and $(1.21)$ \\
$k$, & $n$ & $E_{1,2 n}^{(k)}$ & Error by $(1.14)$ & Error by $(3.10)$ & Error by $(1.21)$ \\
2 & 2 & 1.682364334 & $-5.306866058 e-13$ & $-5.322481309 e-13$ & $-5.299058432 e-13$ \\
3 & 2 & 2.046198632 & 0.00002538068329 & $-2.575717417 e-12$ & $-2.554401135 e-12$ \\
3 & 4 & 1.843393781 & 0.002178484268 & $9.094947018 e-13$ & $2.046363079 e-12$ \\
5 & 4 & 2.295348579 & -0.1173324145 & $-5.820766091 e-11$ & $-2.910383046 e-11$ \\
8 & 3 & 2.777300401 & -0.09936886826 & -0.00000000018917 & -0.00000000018917 \\
8 & 7 & 2.718064292 & -1649334.761 & 0.00048828125 & 0.000244140625
\end{tabular}

It is clear that the errors generated by (1.21) and (3.10) are significantly smaller than those generated by (1.14)

Theorems 1-4 need more precise expressions for $J_{j, k}^{(l)}$ and $K_{j, k}^{(l)}$. Let $y_{j, k}=\cot \frac{j \pi}{k}$ and $z_{j, k}=$ $\csc \frac{j \pi}{k}$. By (1.7), we have

$$
\begin{aligned}
J_{j, k}^{(l)} & =(-\pi)^{l} \sum_{i=0}^{\left[\frac{l+1}{2}\right]} a_{i}^{(l)} y_{j, k}^{l+1-2 i}, \\
K_{j, k}^{(l)} & =(-\pi)^{l} z_{j, k} \sum_{i=0}^{\left[\frac{l}{2}\right]} b_{i}^{(l)} y_{j, k}^{l-2 i},
\end{aligned}
$$

where $a_{i}^{(l)}=0$ for $i>l, a_{0}^{(l)}=l$ ! for $l \geq 0, a_{i}^{(l)}=(l-2 i+2) a_{i-1}^{(l-1)}+(l-2 i) a_{i}^{(l-1)}$ for $0<i \leq l$, and $b_{0}^{(l)}=l$ ! for $l \geq 0, b_{i}^{(l)}=0$ for $l \leq i$, and $b_{i}^{(l)}=(l-2 i+1) b_{i-1}^{(l-1)}+(l-2 i) b_{i}^{(l-1)}$ for $0<i<l$. The following table shows the numerical results and the errors for the calculation of $E_{p, q}^{(k)}$ by using Matlab.

\begin{tabular}{ccccccc} 
& \multicolumn{5}{c}{ Error Comparison $(1.8),(1.9),(1.15)$ and $(3.8)$} \\
$k$ & $p$ & $q$ & $E_{p, q}^{(k)}$ & Error by $(1.8),(1.9)$ & Error by $(1.8),(3.8)$ & Error by $(1.15)$ \\
2 & 2 & 3 & 1.545467399 & $-9.01501096 e-12$ & 0.0000021036317 & $3.476152699 e-11$ \\
3 & 2 & 3 & 1.667985352 & -0.00002538069409 & 0.000003472649784 & $4.462918923 e-11$ \\
3 & 3 & 6 & 1.182692015 & 0.005083129969 & -0.0004898337411 & $3.637978807 e-12$ \\
5 & 2 & 7 & 1.476568495 & 0.1642653878 & 0.003151047946 & 0.0000000001746229827 \\
5 & 5 & 4 & 1.122023699 & -0.006570616384 & -0.0005041710865 & $-2.546585165 e-11$ \\
6 & 6 & 7 & 1.025820391 & -21.54981886 & 1.633777052 & -0.00000008940696716
\end{tabular}


The following table shows the numerical results and the errors for the calculation of $E_{p, q}^{(k)--}$ by using Matlab.

\begin{tabular}{ccccccc}
\multicolumn{6}{c}{ Error Comparison for $(1.10),(1.11),(1.20)$ and $(3.9)$} \\
$k$ & $p$ & $q$ & $E^{(k)-}-{ }_{p}$ & Error by $(1.10),(1.11)$ & Error by $(1.10),(3.9)$ & Error by $(1.20)$ \\
3 & 2 & 3 & 0.7818713769 & 0.0000285532696 & -0.000002604454 & $6.394884622 e-14$ \\
3 & 4 & 5 & 0.9234507369 & 0.002846023297 & -0.000401817273 & $5.456968211 e-12$ \\
5 & 2 & 3 & 0.7583030262 & -0.000110059725 & -0.000004471647 & 0 \\
5 & 2 & 7 & 0.832556 & -0.1638429596 & -0.003101812792 & $-5.820766091 e-11$ \\
5 & 4 & 5 & 0.9211372323 & -0.03276859478 & -0.001861095981 & $-1.455191523 e-11$ \\
7 & 4 & 5 & 0.9207811843 & 0.08452067384 & -0.005106926139 & 0.0000000005820766
\end{tabular}

These tables clearly show that Theorems 1-5 give significantly better accuracy in numerical calculations by Matlab.

\section{References}

[1] P. Flajolet, B. Salvy, Euler sums and contour integral representations, Exp. Math., 7 (1998) $15-35$.

[2] Philippe Flajolet and Bruno Salvy, Euler Sums and Contour Integral Representations, Experimental Mathematics, Vol. 7 (1998), No. 1,15-35

[3] Richard E. Crandall and Joe P. Buhler, On the Evaluation of Euler Sums,Experimental Mathematics, Vol. 3 (1994), No. 4

[4] Mark W. Coffey,On one-dimensional digamma and polygamma series related to the evaluation of Feynman diagrams, Journal of Computational and Applied Mathematics 183 (2005) $84-100$

[5] Kwang-Wu Chen and Minking Eie, Explicit evaluations of extended Euler sums, Journal of Number Theory 117 (2006) 31-5

\section{Received: August 11, 2013}

